\title{
Die Jodometrie von Hypophosphiten und Hypophosphaten.
}

\author{
Vou E. Rupp und stud. Finck.
}

(Eingegangen den 12. XI. 1902.)

In den Berichten der Deutschen Chemischen Gesellschaft ${ }^{1}$ ) hatten wir unlängst die Titrierbarkeit der phosphorigen Săure dargethan. Nachstehend soll dasselbe für die unterphosphorige Săure, deren Salze medizinische Verwertung finden und für die von Salzer entdeckte Unterphosphorsăure dargethan werden.

Wie l. c. ausgeführt, ist das Jodabsorptionsvermögen der phosphorigen Säure in saurer und neutraler Lösung ein tiberaus geringes, während in bikarbonatalkalischer Lösung die Reaktion innerhalb Stundenfrist quantitativ beendet ist. Als ganz entgegengesetzt erwies sich das Verhalten der unterphosphorigen Säure, indem deren Oxydationsgeschwindigkeit gerade in alkalisierter Lösung die allerträgste ist, in angesăuerter Lösung hingegen im Verhältnis des Dissociationsgrades der zugeführten Säure ansteigt. Die Oxydation vollzieht sich also quantitativ um so rascher je weitgehender die Dissociation der unterphosphorigen Säure selbst zurückgedrängt wird. Als Grund des verschiedenen Verhaltens der beiden Säuren ist demnach, vorbehältlich einer theoretisch-experimentellen Untersuchung, der Umstand zu betrachten, dass die Oxydation der unterphosphorigen Săure eine Reaktion der undissociierten $\mathrm{H}_{8} \mathrm{PO}_{2}$-Moleküle darstellt, während bei der phosphorigen Săure eine Ionenreaktion

vo!'jiegt.

$$
\mathrm{HPO}_{\mathrm{B}} "+\mathrm{O}=\mathrm{HPO}_{4}
$$

Die Versuche betrafen als das pharmazeutisch wichtigste Hypophosphit zunächst das

\section{Calcium hypophosphorosum.}

Denkt man sich die Oxydation der unterphosphoriven Säure in den zwei Phasen:

$$
\begin{aligned}
& \text { 1. } \mathrm{H}_{8} \mathrm{PO}_{2}+\mathrm{O}=\mathrm{H}_{8} \mathrm{PO}_{8} \\
& \text { 2. } \mathrm{H}_{8} \mathrm{PO}_{3}+\mathrm{O}=\mathrm{H}_{3} \mathrm{POO}_{4}
\end{aligned}
$$

verlaufend, so berechnet sich der Jodverbrauch für Phase 1 nach dem Ansatze :

$$
\begin{aligned}
& \mathrm{I}_{8} \mathrm{PO}_{2}=2 \mathrm{~J} \\
& \frac{\mathrm{H}_{\mathrm{B}} \mathrm{PO}}{20000}=0,0033 \mathrm{~g}=1 \mathrm{ccm} \mathrm{n} / 10 \mathrm{~J} .
\end{aligned}
$$

1) Ber. d. d. chem. Ges. 35, 3691. 
Desgleichen für Phase 2. Für Phase $1+2$ also:

$$
\frac{\mathrm{H}_{8} \mathrm{PO}_{2}}{40000}=0,00165 \mathrm{~g}=1 \mathrm{ccm} \mathrm{n} / 10 \mathrm{~J} \text {. }
$$

Der Jodverbrauch des Calciumsalzes stellt sich dementsprechend auf:

bezw.

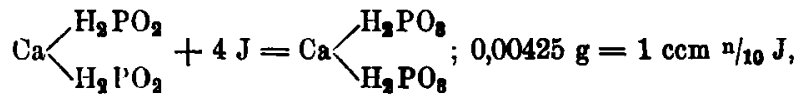

$$
\mathrm{Ca}\left\langle{ }_{\mathrm{H}_{2} \mathrm{PO}_{2}}^{\mathrm{H}_{2} \mathrm{PO}_{2}}+8 \mathrm{~J}=\mathrm{Ca}\left\langle\mathrm{H}_{2} \mathrm{HO}_{2} \mathrm{H}_{2} \mathrm{PO}_{4} ; 0,002125 \mathrm{~g}=1 \mathrm{ccm} \mathrm{n} / 30 \mathrm{~J}\right. \text {. }\right.
$$

$18,23 \mathrm{~g}$ eines gut krystallisierten Präparates von Calciumhypophosphit wurden in $1500 \mathrm{ccm}$ Wasser gelöst, so dass also $1 \mathrm{ccm}$ $0,012153 \mathrm{~g}$ Hypophosphit mit dem theoretischen Jodwerte 2,859 ccm bezw. $5,719 \mathrm{ccm}{ }^{n} / 10-$ Losung enthielt.

$5 \mathrm{ccm}$ dieser Lösung wurden mit $1 / 2-1 \mathrm{~g}$ Mononatriumkarbonat und je $50 \mathrm{ccm}$ Jodlösung stehen gelassen. Durch Rücktitration mit Thiosulfatlösung konnte konstatiert werden, dass

$\begin{array}{ccccccc}\text { nach } & 11 / 8 & \text { stündiger } & \text { Reaktionsdauer } & 1,8 \mathrm{~cm} & \mathrm{~J} \\ n & 2 & n & n & 1,88 & n \\ n & 15 & n & n & 3,1 & n\end{array}$

verbraucht worden waren.

Höchst uberraschender Weise war also die stark reduzierende Wirkung der unterphosphorigen Säure unter diesen Bedingungen so gut wie nicht zur Bethätigung gelangt.

Wurde ohne Neutralisationsmittel oder mit Zusătzen solcher Sauren gearbeitet, welche die unterphosphorige Săure nicht in Freiheit zu setzen vermogen, so fand ebenfalls nur eine geringe Reaktion statt.

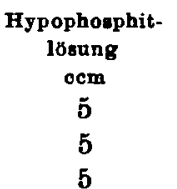

Jodloung
angewandt
$\mathrm{ccm}$
50
50
50

50

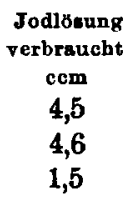

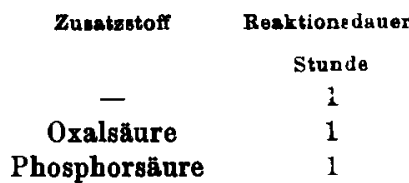

Es schien, dass der infolge spurweiser Oxydation gebildete Jodwasserstoff die Veranlassung zu dem immerhin etwas gesteigerten Reaktionsvorgange war, indem jener aus einem Teil des Hypophosphits die Săure selbst in Freiheit setzte, und dass diese nun leichter der Oxydation unterlag. Diese Annahme als richtig vorausgesetzt, musste sich der Jodverbrauch in stark mineralsaurer Lösung erheblich steigern. Die nachfolgenden in schwefelsaurer Lösung ausgeführten Versuche verliefen denn auch thatsăchlich in diesem Sinne. 
E. Rupp a. Finck: Hypophosphite und Hypophosphate.

$\begin{array}{ccc}\begin{array}{c}\text { Jodlösung } \\ \text { angewandt } \\ \text { ccm }\end{array} & \begin{array}{c}\mathbf{n} / 10 \text { Thiosulfat } \\ \text { verbrauch } \\ \text { cem }\end{array} & \begin{array}{c}\text { Jod- } \\ \text { verbrauch } \\ \text { ccm }\end{array} \\ 50 & \mathbf{4 3 , 4 0} & \mathbf{6 , 6 0} \\ 50 & \mathbf{4 2 , 6 6} & \mathbf{7 , 3 7} \\ 50 & \mathbf{3 8 , 9 5} & \mathbf{1 1 , 0 5} \\ 50 & \mathbf{3 9 , 2 0} & \mathbf{1 0 , 8 0} \\ 50 & \mathbf{3 7 , 0 0} & \mathbf{1 3 , 0 0} \\ 50 & \mathbf{3 7 , 0 5} & \mathbf{1 2 , 9 5} \\ 50 & \mathbf{3 4 , 5 0} & \mathbf{1 5 , 5 0}\end{array}$

$\begin{array}{cc}\text { Znsatratoff } & \text { Zeitdauer } \\ \text { cem } \mathrm{H}_{2} \mathrm{SO}_{4} & \text { stunde } \\ 5 & 11 / 4 \\ 5 & 11 / 4 \\ 10 & 11 / 4 \\ 10 & 11 / 4 \\ 50 & 11 / 4 \\ 50 & 11 / 4 \\ 5 & 2\end{array}$

Die Versuchsreihe wurde mit einer neuen Lösung fortgesetzt, die $0,009831 \mathrm{~g}$ Calciumhypophosphit im Knbikcentimeter enthielt. $10 \mathrm{ccm}$ derselben wurden mit je $25 \mathrm{ccm} n / 10 \mathrm{Jod}$ und $5 \mathrm{ccm}$ verdünnter Schwefelsäure 15 Stunden stehen gelassen and alsdann zurucktitriert. Es waren nun an Jod verbraucht worden

1. $23,28 \mathrm{ccm}$

2. 23,30 "

3. 23,29 ,

im Mittel also 23,29 $\mathrm{ccm}$.

Nach 16-20 Stunden Stehens wurde ein nur unwesentlich erhöhter Jodverbrauch notiert.

Es musste daher scheinen, dass eine zu einheitlichen konstanten Werten führende Reaktion erfolgt war.

Berechnet man den Jodverbrauch auf die angewandte Hypophosphitmenge, so zeigt sich, dass die Oxydation im Sinne der Gleichung vor sich gegangen war, die oben als 1 . Phase bezeichnet worden war.

1 ccm $\mathrm{n} / 10 \mathrm{~J}=0,00425$ g $\mathrm{C2}_{2}\left(\mathrm{H}_{2} \mathrm{PO}_{2}\right)_{2}$

23,29 n $\mathrm{n} / 10$ n $=0,09898$, Angewandt:

Gefunden:

$$
0,09831 \mathrm{~g}=100 \% \quad 0,09898 \mathrm{~g}=100,68 \% \text {. }
$$

Notwendigerweise mussten die titrierten Løsungen nun phosphorige Săure enthalten. Da aber letztere, wie 1 . c. behandelt, in bikarbonatalkalischer Losung mit Jod titrierbar ist, so mussten die Titrationsflüssigkeiten nach der Alkalisierung zu einer nochmaligen Jodaufnahme gleich hohen Betrags unter Oxydierung der phosphorigen Säure zu Phosphorsäure betähigt sein.

Letztaufgeführte drei Proben wurden also mit Mononatriumkarbonat im kleinen Ueberschusse nentralisiert, mit je $30 \mathrm{ccm}$ Jod. lösung versetzt und nach $1 \frac{1}{2}$ Stunden zuräcktitriert.

Der Jodverbrauch betrug:

1. $22,90 \mathrm{ccm}$

2. 22,92 ,

3. 22,90 ,

im Mittel 22,90 ccm. 
Diese Werte den in Phase I erhaltenen hinzuaddiert, ergeben im Mittel 46,19 ccm. Hierauf berechnet ist das Resultat folgendes:

Angewandt:

Gefunden:

$0,09831 \mathrm{~g} \mathrm{Ca}\left(\mathrm{H}_{2} \mathrm{PO}_{2}\right)_{2}=100 \% \quad 0,098153 \mathrm{~g} \mathrm{Ca}\left(\mathrm{H}_{2} \mathrm{PO}_{2}\right)_{2}=99,84 \underset{o}{\mathrm{o}}$, da $1 \mathrm{ccm} \mathrm{n} / 10 \mathrm{~J}=0,002125 \mathrm{~g}$ Calciumhypophosphit.

In genauester Weise giebt also der Summenwert einer Ioppeltitration die vorhandene Hypophosphitmenge an.

Um das angewandte Präparat von Hypophosphit auch durch eine gravimetrische Analyse zu charakterisieren, wurde dessen Calciumgehalt bestimmt.

$25 \mathrm{ccm}$ der Lősung mit oxalsaurem Ammonium behandelt lieferten geglüht

$$
\begin{aligned}
& 0,0812 \text { g } \mathrm{Ca} O \\
& 0,0813 ",
\end{aligned}
$$

im Mittel $0,08125 \mathrm{~g}$, welche $0,2466 \mathrm{~g}$ Calciumbypophosphit $=100,36 \%$ der angewandten Menge von 0,2457 g entsprechen.

Aus der Titration in saurer Lösung allein werden deshalb et was $u$ hohe Resultate gefunden, weil spurweise die gebildete phosphorige Säure auch in der schwefelsauren Lösung weiter oxydiert wird.

Das Anwachsen dieses Betrags mit der Reaktionsdauer erhellt aus folgender Reihe:

$10 \mathrm{ccm}$ Hypophosphitlösung mit Schwefelsäure und mit $25 \mathrm{ccm} \mathrm{n/10} \mathrm{Jod}$ 7 Stunden stehen gelassen, hatten $22,95 \mathrm{ccm}$ Jod verbraucht. 15 Stunden stehen gelassen waren $23,30 \mathrm{ccm}$ Jod verbraucht worden. $Z u$ der noch 7 Stunden titrierten Probe wurden abermals $30 \mathrm{ccm}$ Jod gegeben und weitere 15 Stunden stehen gelassen; es waren dann weitere $0,92 \mathrm{ccm}$ Jod hinzuverbrancht worden. Den Jodzusatz wiederholt und nochmals 48 Stunden stehen gelassen, hatte die Lösung abermals $1,63 \mathrm{ccm}$ Jod verbraucht.

Es waren also bei dieser aussergewöhnlich langen Reaktionsdauer $11 \%$ der gebildeten phosphorigen Saure der Weiteroxydation unterlegen, bei 15 stündiger Einwirkung jedoch nur $1 \%$.

Die ermittelten Verbältnisse wurden nochmals bestätigt an einem anderen Calciumhypophosphitpräparate. Dasselbe war im Verhältnis $15,218 \mathrm{~g}=1000 \mathrm{ccm}$ in Wasser gelöst worden.

Die Kalkbestimmung in $25 \mathrm{ccm}=0,3804 \mathrm{~g}$ Substanz, lieferte an Calciumsulfat

$$
\begin{aligned}
& \text { 1. } 0,3042 \mathrm{~g} \\
& \text { 2. } 0,3038 \text { " }
\end{aligned}
$$

im Mittel $0,304 \mathrm{~g} \mathrm{Ca} \mathrm{SO})_{4}=0,38 \mathrm{Ca}\left(\mathrm{H}_{2} \mathrm{PO}_{2}\right)_{2}=99,89 \%$ der angewandten Menge.

Zwei Proben standen 15 Stunden mit $5 \mathrm{ccm}$ Schwefelsãure und $50 \mathrm{ccm}$ n/10 Jodlösung. 
E. Rupp u. Finck: Hypophosphite und Hypophosphate.

$\begin{array}{cc}\mathrm{n} / \mathrm{so} \text { Thiosulfalverbrauch: } & \text { Jod verbrauch: } \\ \text { 1. } 13,00 \mathrm{ccm} & 37,00 \mathrm{ccm} \\ \text { 2. } 12,93 & 37,07\end{array}$

Mit Natriumbikarbonat neutralisiert, mit weiteren Mengen von Jodlösung versetzt und nach $11 / 2$ Stunden zarücktitriert war das Ergebnis folgendes:

n/10 Jod angewandt n/10 Thiosulfatrerbrauch Jodverbranch

1. $50 \mathrm{ccm}$

2. 50 ,

1.

$34,75 \mathrm{ccm}$

$\frac{+37,00}{71,75 \mathrm{ccm}}$
$15,25 \mathrm{ccm}$

15,32 »

2.

$34,68 \mathrm{ccm}$
$34,75 \mathrm{ccm}$

34,68 r

$=100,11 \%$. Theoretischer Jodwert $35,85 \mathrm{ccm}$ bezw. $71,70 \mathrm{ccm} \mathrm{n/10} \mathrm{Jod.}$

Nach 6 stündiger Reaktionsdauer in saurer Lösung stellte sich das Verhältnis für $10 \mathrm{ccm}$ wie folgt :

$n / 10$ Jod angewandt

$50 \mathrm{ccm}$

Nach 7 Stunden:

$\mathrm{n} / 10$ Jod angewandt

$50 \mathrm{ccm}$

50 n

50

50 , n/10 Thiosulfatverbrauch

$14,43 \mathrm{ccm}$

${ }_{10}$ Thiosulfatverbrauch

$14,35 \mathrm{ccm}$

14,35 ;

14,25 ,

14,30 ,

$14,05 \mathrm{ccm}$

14,17 ,

$12,53 \mathrm{ccm}$

12,50 , n/10 Jodverbrauch

$35,57 \mathrm{ccm}$.

Nach 8 Stunden:

$50 \mathrm{ccm}$

50 "

Nach 24 Stunden:

$50 \mathrm{ccm}$

50 ,

In einem anderen Versuche hatte die Reaktionsdauer in saurer Lösung nur 5 Stunden betragen,

n/10 Jod angewandt

$50 \mathrm{ccm}$

n/10 Thiosulfatverbrauch

n/10 Jodverbrauch

$14,65 \mathrm{ccm}$

$35,35 \mathrm{ccm}$,

dann war mit Mononatriumkarbonat und Jod versetzt und nach 11/2 Stunden wieder titriert worden:

$n / 10 \mathrm{Jod}$ angewandt $50 \mathrm{ccm}$

n/10 Thiosulfaterbrauch $14,50 \mathrm{ccm}$ n/10 Jodverbranch $35,50 \mathrm{ccm}$.

Also im ganzen verbraucht $70,85 \mathrm{ccm}=(2 \times 35,42 \mathrm{c} \mathrm{cm})=98,9 \%$. Wie aus der Differenz 35,35 gegenüber 35,50 ersichtlich, hatte nunmehr die zweite Titration ein höheres Resultat ergeben, indem eine gewisse Quantität der in saurer Lösung wegen zu kurzer Reaktionsdauer unoxydiert gebliebenen unterphosphorigen Säure i. der neutralisierten Lösung nachoxydiert wurde. 
Die richtigen Versuchsbedingungen für die Jodometrie der unterphosphorigen Säure sind dahin zusammenzufassen, dass zunächst in schwefelsaurer Lösung mit überschüssigem Jod ca. 10 Stunden stehen gelassen wird, hierauf misst man den Jodüberschuss zurück, neutralisiert mit einem kleinen Ueberschusse von Mononatriumkarbonat, setzt abermals Jodlösung $\mathrm{zn}$ und misst nach $1-1^{1 / 2}$ stündigem ruhigen Stehen zurick.

Es kann die erstmalige Rücktitration umgangen werden, indem die viel überschüssiges Jod enthaltende Lösung nach 12-15 Stunden direkt neutralisiert und nach weiteren $1 \frac{1}{2}$ Stunden mit Thiosulfat titriert wird. Wir hatten zuerst gezögert, diesen Weg einzuschlagen, da Jodverluste bei der Neutralisation mit Bikarbonat bezw. der damit verbundenen Kohlendioxyd-Entwickelung unvermeidlich schienen.

Nachstehende Parallelversuche zeigen indes, dass auf diese einfachere Weise, wenn auch nicht so absolut konstante, doch wohl brauchbare Werte erzielbar sind.

$10 \mathrm{ccm}$ einer Hypophosphitlösung von $3,7696 \mathrm{~g}$ in $250 \mathrm{ccm}$ Wasser hatten nach 12 stündigem Stehen mit $5 \mathrm{ccm}$ verdünntem $\mathrm{H}_{2} \mathrm{SO}_{4}$ and 50 (cm $\mathrm{n} / 10 \mathrm{~J}$ ron letzterem

$$
\begin{aligned}
& \text { 1. } 35,90 \mathrm{ccm} \\
& \text { 2. } 35,97 \text { n }
\end{aligned}
$$

verbraucht, hierauf neutralisiert und nochmals mit $50 \mathrm{ccm} \mathrm{n/10} \mathrm{Jod} \mathrm{versetzt,}$ waren nach 11/2 Stunden nochmals an Jod verbraucht worden

1. 35,02 in Summa $70,92 \mathrm{ccm}$

2. $34,90, \# 70,87$,

Gefunden:

Angewandte Hypophosphitmenge:
1. $0,1507 \mathrm{~g}=100_{\%}^{\circ}$
$0,1507 \mathrm{~g}=100 \%$.
2. 0,1506 " $=99,7$,

$5 \mathrm{ccm}$ derselben Hypophosphitlösung wurden mit $5 \mathrm{ccm}$ verdünntem $\mathrm{H}_{2} \mathrm{SO}_{4}$ und $50 \mathrm{ccm} \mathrm{n} / 10$ Jod 12 Stunden stehen gelassen, dann durch Natriumbikarbonat mit der Vorsicht neutralisiert, dass das Bikarbonat langsam in den möglichst schräg gehaltenen Kolben eingetragen wurde. Nach weiteren 11/2 Stunden war mit Thiosulfat zurücktitriert worden.

Das Ergebnis dreier Proben war:

$\begin{array}{ccc}\text { n/10 Thiosulfatverbrauch } & \text { Jodverbrauch } & \text { Gefundene Hypophosphitmenge } \\ 14,50 \mathrm{ccm} & 35,50 \mathrm{ccm} & 100,01 \\ 14,85 n & 35,15 n & 99,13 n \\ 14,72 n & 35,28 n & 99,49\end{array}$

Pro praxi wăre die Titrationsvorechrift für Calcium hypophosphorosum demnach wie folgt zu gestalten: Man löse im $100 \mathrm{ccm}$ Kolben 1,5 $\mathrm{g}$ des Präparates in Wasser auf und fülle bis zur Marke an. Sodann sptile man $5 \mathrm{ccm}$ der Lösung mit etwas Wasser in ein 
Glasstöpselglas von ca. $200 \mathrm{ccm}$ und gebe $50 \mathrm{ccm}$ n/10 Jodlösung nebst $5 \mathrm{ccm}$ verdünnte Schwefelsäure hinzu. Nachdem man 12-15 Stunden an dunklem Orte bei Zimmertemperatur stehen gelassen, wird portionenweise mit Natriumbikarbonat versetzt, derart, dass nach beendeter Kohlensaureentwickelung nochmals ca. $1 / 2$ g hinzugefügt wird. Nach weiteren zwei Stunden Stehens wird mit $\mathbf{n} / 10$ Thiosulfat zurücktitriert und sollen hiervon nicht mehr als $15,5 \mathrm{ccm}$ erforderlich sein. (Theoretischer Verbrauch $=14,7 \mathrm{ccm}$ Thiosulfat $=35,3 \mathrm{ccm}$ Jod).

Wir haben die Erfahrung gemacht, dass gute Handelspräparate von weisser Farbe und krystallinischer Beschaffenheit diesen Anforderungen stets genügten, während hygroskopische, missfarbige und trüblösliche Marken beträchtliche Unterwerte ergaben.

Jodometrie der freien Säure: Das zur Untersuchung vorliegende sirupöse Präparat wurde zunächst einer gravimetrischen Analyse in der Weise unterworfen, dass einmal die direkt vorhandene und ein zweites Mal die nach der Oxydation mit chlorsaurem Kalium und Salzsăure gebildete Gesamtphosphorsäure bestimmt wurde. Von der durch Oxydation entstandenen Phosphorsäure war wohl mit aller Wahrscheinlichkeit anzunehmen, dass sie nicht allein aus unterphosphoriger Säure, sondern zu einem gewissen Prozentsatze auch aus phosphoriger Säure hervorgegangen war, die ja schon durch den Luftsauerstoff mit grösster Leichtigkeit aus der unterphosphorigen Säure sich bildet ${ }^{1}$ ). Wir haben es unterlassen gravimetrisch festzustellen, inwieweit dies der Fall war, da eine direkte 'Trennungsmethode der beiden Säuren nicht vorzuliegen scheint. Jodometrisch hingegen war anzunehmen, dass die Differenzen der beiden Titrationen in saurer und alkalischer Lösung jedenfalls einen annähernden Schluss auf das Quantitätsverhältnis der beiden Săuren gestatten würden.

$10 \mathrm{ccm}$ der sirupঠsen Săure wurden auf $1000 \mathrm{ccm}$ verdünnt und dann $100 \mathrm{ccm}$ dieser Lösung mit Kaliumchlorat und Salzsäure oxyđiert. Daraus wurden erhalten:

$$
\begin{aligned}
& \text { 1. } 0,6650 \mathrm{~g} \mathrm{Mg}_{2} \mathrm{P}_{2} \mathrm{O}_{7} \\
& \text { 2. } 0,6636,
\end{aligned}
$$

im Mittel $0,6643 \mathrm{~g}$ pro $1 \mathrm{ccm}$ sirupőser Säure.

Die Bestimmung der direkt vorhandenen Phosphorsüure wurde mit 5 ccm des ursprünglichen Präparates ausgeführt und lieferte:

$$
\begin{aligned}
& \text { 1. } 0,0096 \text { g } \mathrm{M}_{2} \mathrm{P}_{2} \mathrm{O}_{7} \\
& \text { 2. } 0,0082 n " n
\end{aligned}
$$

im Mittel $0,0089 \mathrm{~g}$, also für $1 \mathrm{ccm}=\frac{0,0089}{5}=0,0017 \mathrm{~g}$.

1) Rammelsberg, Berl. Ber. 1, 185. 
Aus der Differenz

$$
\begin{aligned}
& 0,6643 \mathrm{~g} \mathrm{Mg} \mathrm{M}_{2} \mathrm{O}_{7} \\
& \frac{0,0017 n}{0,6626 \mathrm{~g} \mathrm{Mg} \mathrm{Mg}_{2} \mathrm{O}_{7}}
\end{aligned}
$$

berechnet sich alsdann ein Gehalt von $0,3939 \mathrm{~g}$ Unterphosphorigsäure für einen Kubikzentimeter sirupöser Säure - mit dem Vorbehalte, dass hierron ein gewisser Prozentsatz als phosphorige Säure anzusprechen ist.

Die nachstehenden Titrationon wurden mit je $10 \mathrm{ccm}$ obiger I.ösung $1: 100$ ansgeführt, die $0,03939 \mathrm{~g}$ Unterphosphorigsäure entsprechen. Die Reaktionsdauer für die Säurelösung war auf $12-15$ Stunden, für die alkalisierte Lösung auf 1 Stunde gestellt. Die angewandte Jodmenge war $25 \mathrm{ccm}$.

\begin{tabular}{cccc} 
n/10 Thiosulfatverbrauch & n/10 Jodverbrauch & \multicolumn{2}{c}{ Zusatzstoff } \\
$14,02 \mathrm{ccm}$ & $10,98 \mathrm{ccm}$ & $5 \mathrm{ccm}$ verd. $\mathrm{H}_{2} \mathrm{SO}_{4}$ \\
$14,07 \%$ & $10,93 \%$ & $5 \% "$
\end{tabular}

Neutralisiert mit Mononatrinmkarbonat und nochmals Jod zugesetzt n/10 Jod angewandt $\quad n / 10$ Thiosulfatverbrauch n/10 Jodverbrauch
1. $25 \mathrm{ccm}$ $12,90 \mathrm{ccm}$ $12,10 \mathrm{ccm}$
2. 25 ,
12,85 " $12,1 \overline{0}$,

Demnach in Summa verbraucht $23,08 \mathrm{ccm} \mathrm{n}_{10} \mathrm{Jod}=0,03808 \mathrm{~g}$ Unterphosphorigsäure $=96,67 \stackrel{\sim}{*}$ der gravimetrisch gefundenen Mengen von $0,03939 \mathrm{~g}, \mathrm{da}$

$$
\begin{aligned}
& 1 \mathrm{H}_{8} \mathrm{PO}_{2}=4 \mathrm{~J} \\
& { }_{40000}^{\mathrm{H}_{8} \mathrm{PO}_{2}}=0,00165 \mathrm{~g}=1 \mathrm{ccm}^{\mathrm{n}}{ }_{10} \mathrm{~J} \text {. }
\end{aligned}
$$

Wie er'sichtlich, verrăt sich hier das geänderte Verhältnis schon dadurch, dass nunmehr die Titration in saurer Lösung einen geringeren Wert ergeben hatte, als in alkalischer Lösung, während bei reiner Hypophosphitlösung stets der erst erhaltene Wert der höhere war, weil schon in saurer Lösung etwas gebildete phosphorige Säure mitoxydiert wird.

Es ergiebt sich daraus unzweideutig, dass das angewandte Präparat von unterphosphoriger Säure thatsächlich phosphorige Säure enthielt, und zwar in einer Menge, die sehr annähernd der Differenz $12,15-10,95=1,2 \mathrm{ccm}$ entsprechen muss. Diese $1,2 \mathrm{ccm}$ den verbrauchten 23,08 hinzuaddiert giebt $24,2 \mathrm{ccm}=101,37 \%$.

Das Verhältnis von $\mathrm{H}_{3} \mathrm{PO}_{2}: \mathrm{H}_{3} \mathrm{PO}_{3}$ lässt sich hieraus wie folgt annähernd berechnen: $10,98 \mathrm{ccm} / 10 \mathrm{~J}=0,03623 \mathrm{~g}$ Unterphosphorigsäure $\left(\mathrm{H}_{8} \mathrm{PO}_{2}=2 \mathrm{~J}\right)=91,98 \%(0,03939 \mathrm{~g}=100 \%)$, so dass als phosphorige Säure anzusprechen sind $=8,02 \%$.

Wie mehrfach erwähnt, kann dieses Resultat nur als ein ungefähres, wie die Ausführung zeigt nm etwa $1 \%$ differierendes betrachtet werden. Qualitativ konnte mittelst Baryumchlorid der 
Nachweis geftihrt werden, dass die unterphosphorige Săure phosphorige Sïure enthalten hatte, indem eine Probe mit Ammoniak neutralisierter, von Phosphorsäure durch Magnesiamixtur befreiter Säure nach der Filtration mit Chlorbaryumlösung versetzt, einen Niederschlag von Baryumphosphit gab. Bei Abwesenheit von phosphoriger Suure bätte die Lösung klar bleiben müssen.

\section{Unterphosphorsāure.}

Die Titrimetrie der Unterphosphorsäure war praktisch genommen nur von untergeordneter Bedeutung, theoretisch bot sie Interesse deshalb, weil deren jodometrische Bestimmbarkeit in gewissem Grade konstitutionsbeweisend sein musste.

Die Säure ist bekanntlich auffassbar als extramolekulares Anhydrid von 1 Mol. phosphoriger Säure und 1 Mol. Phosphorsäure

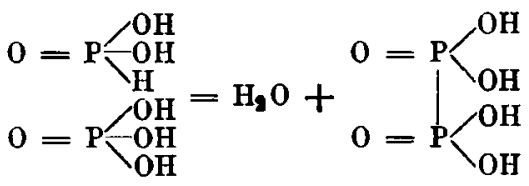

War die Säure im umgekehrten Sinne wieder hydratisierbar, so musste sie jodometrisch sich verhalten wie die phosphorige Săure, also 1 Mol. musste 2 Atomen Jod entsprechen:

$$
\begin{aligned}
& \mathrm{H}_{4} \mathrm{P}_{2} \mathrm{O}_{6}=2 \mathrm{~J} \\
& \frac{\mathrm{H}_{4} \mathrm{P}_{2} \mathrm{O}_{6}}{20000}=0,0081 \mathrm{~g}=1 \mathrm{ccm} \mathrm{n} / 10 \text { Jodlösung. }
\end{aligned}
$$

Es wurde versucht nach der Vorschrift von Salzer ${ }^{1}$ ) ein Präparat der Unterphosphorsäure zunächst ans der sogenannten phosphatischen Saure zn gewinnen - jener sirupösen Flüssigkeit, welche beim $\mathrm{Zu}$ fliessen von Phosphor in feuchter Luft gewonnen wird. Die benützte phosphatische Säure lieferte jedoch in keiner der Krystallisationen, welche nach der Neutralisation daraus gewonnen waren, isolierbare Mengen des sauren Natriumsalzes der Unterphosphorsäure, $\mathrm{HNaPO}_{\mathbf{a}}+$ $3 \mathrm{H}_{2} \mathrm{O}$.

Wir suchten dasselbe nun nach der Methode von Corne ${ }^{8}$ ) darzustellen. Zu diesem Zwecke wurden $20 \mathrm{~g}$ Cuprinitat in Wasser gelöst, die Lösung im Wasserbade auf $70^{\circ}$ erwärmt und der Kolben mit einem Steigrohr versehen. Von Zeit zu Zeit wurde ein Stúckchen Phosphor eingetragen und damit bis zur Entfärbung fortgefahren.

1) Salzer, Ann. d. Chem. 187, 322; 194, 28; 211, 1.

8) Chem. Centralbl. 1882, 611. 
Nach dem Erkalten wurde die Lobsung in zwei Teile geteilt, ein Teil mit Natriumkarbonat gesättigt und dem zweiten Teil hinzugefügt. Auch so gelang es nicht, zu einem einheitlichen Salz der Unterphosphorsäure zu gelangen.

Mit Leichtigkeit hingegen ist ein solches darstellbar durch Oxydation von Phosphor mit Silbernitrat nach Jul. Philipp'). In die Lösung von $6 \mathrm{~g}$ Silbernitrat in $100 \mathrm{ccm}$ Salpetersäure (Sp. G. 1,2) und $100 \mathrm{ccm}$ Wasser, welche ich in einem sehr geräumigen Kolben (dessen Oeffnung durch einen aufgesetzten kleinen Trichter verengt war) auf dem Wasserbade stark erwärmte, wurden 8-9 g Phosphor eingetragen. Sobald die eintretende heftige Reaktion nachgelassen, wurde das Gefäss vom Wasserbade heruntergenommen und die Flüssigkeit nach dem Erkalten von dem uberschüssigen Phosphor durch Filtration getrennt; letzterem ist meist etwas auskrystallisiertes unterphosphorsaures Silber beigemengt, welches durch kurzes Erwarrmen mit neuen Mengen Salpetersäure entzogen wurde.

Wahrend des Erkaltens der Flüssigkeit krystallisiert etwas unterphosphorsaures Silber aus, während der grosste Teil desselben durch Neutralisation der Lơsung mit Ammoniak gefällt wurde. Wenn die Flüssigkeit durch Ammoniak dem Punkte der Neutralisation nahe gebracht ist, mischen sich dem Niederschlag kleine Mengen phosphorigsauren Silbers bei, welche eine nachträgliche Gelb-resp. Braunfärbung verursachen. Nachdem alles Silber aus der Flüssigkeit gefällt worden, waren in derselben noch beträchtliche Mengen von Unterphosphorsïure vorhanden, welche durch Zusatz von neuen Mengen Silbernitrats, Ansäuren mit Salpetersäure und Neutralisation mit Ammoniak erhalten wurden. Um unterphosphorsaures Natrium zu erhalten, wurde der Niederschlag mit Chlornatrium behandelt und von dem entstandenen Chlorsilberniederschlag abfiltriert. Die Flüssigkeit wurde eingeengt und zur Krystallisation beiseite gestellt. Die Krystalle wurden hierauf aus ganz verdünnter reiner Natronlauge umkrystallisiert, um neutrales unterphosphorsaures Natrium za erhalten.

\section{Analyoe:}

$0,6346 \mathrm{~g}$ bei $105^{\circ}$ bis zur Gewichtskonstanz getrocknet verloren $0,2646 \mathrm{~g}$ an Gewicht. Mit den restierenden $0,37 \mathrm{~g}$ wurde nach der Oxydation eine Phosphorsüurebestimmung ausgeführt, welche $0,3245 \mathrm{~g} \mathrm{Mgg}_{9} \mathrm{P}_{2} \mathrm{O}_{7}$ ergab.

\section{Gefunden:}

$\mathrm{H}_{2} \mathrm{O} \quad 41,69 \%$

$\mathrm{Na}_{4} \mathrm{P}_{9} \mathrm{O}_{6} \quad 57, \mathbf{5 8}$,

$2,0339 \mathrm{~g}$ des Salzes wurden in $250 \mathrm{ccm}$ Wasser gelöst.

Berechnet:

$$
10 \mathrm{H}_{2} \mathrm{O} \quad 41,86 \%
$$$$
\mathrm{Na}_{4} \mathrm{P}_{2} \mathrm{O}_{6} \quad 58,14, .
$$

1) Berl. Ber. 16, 749 . 
E. Rupp u. Finck: Hypophosphite und Hypophosphate.

1. $25 \mathrm{ccm}$ dieser Lösung mit Natriumbikarbonat und $20 \mathrm{ccm} \mathrm{n} / 10 \mathrm{Jod}-$ lösung 1 Stunde bezw. 5 und 15 Stunden stehen gelassen:

n/10 Thiosulfat-

verbrauch

$15,73 \mathrm{ccm}$

13,08 "

13,07 , n/10 Jodverbrauch

$4,27 \mathrm{ccm}$

6,92

6,97 ?
Zeitdauer

1 Stunde

5

15 Stunden
$73,19 \%$

2. $25 \mathrm{ccm}$ mit Natriumkaliumtartrat und $20 \mathrm{ccm} \mathrm{n} / 10$ Jodlösung 1 Stunde stehen gelassen:

$\begin{array}{cc}\text { n/10 Thiosulfatverbrauch } & \text { n/10 Jodverbrauch } \\ 17,94 \mathrm{ccm} & 2,06 \mathrm{ccm} .\end{array}$

3. $25 \mathrm{ccm}$ mit Natriumkaliumtartrat und $20 \mathrm{ccm} \mathrm{n} / 10 \mathrm{Jod} 15$ Stunden stehen gelassen:

n/10 Thiosulfatverbrauch
$15,99 \mathrm{ccm}$
15,81
n/10 Jodverbrauch $4,01 \mathrm{ccm}$ 4,19 ".

4. $25 \mathrm{ccm}$ mit $5 \mathrm{ccm}$ verdünnter Schwefelsäure 15 Stunden gestanden:

n/10 Thiosulfatverbrauch $19,87 \mathrm{ccm}$ n/10 Jodverbrauch

$0,13 \mathrm{ccm}$.

5. Wir erwärmten $10 \mathrm{ccm}$ auf dem Wasserbade eine Stunde lang mit $5 \mathrm{ccm}$ verdünnter Schwefelsäure, dann wurde mit Mononatriumkarbonat neutral gemacht und 1 Stunde stehen gelassen:

n/10 Jod angewandt n/10 Thiosolfat- n/10 Jodrerbrauch
$20 \mathrm{ccm}$
$17,8 \mathrm{ccm}$
$2,2 \mathrm{ccm}$
$58,13 \%$.

6. $25 \mathrm{ccm}$ mit $5 \mathrm{ccm} \mathrm{H}_{2} \mathrm{SO}_{4} 1$ Stunde gekocht, mit Natriumbikarbonat neutral gemacht und mit $25 \mathrm{ccm}$ Jod 1 Stunde stehen gelassen.

$\begin{array}{ccc}\text { n/10 Thiosulfatrerbrauch } & \text { n/10 Jodverbrauch } & \\ 17,91 \mathrm{ccm} & 7,09 \mathrm{ccm} & 74,98 \% \\ 18,75 n & 6,25 \% & -\end{array}$

Eine weitere Lösung des Salzes war hergestellt worden durch Lösen von $1,5104 \mathrm{~g}$ Salz in $200 \mathrm{ccm}$ Wasser.

7. $20 \mathrm{ccm}$ der Lösung wurden mit $10 \mathrm{ccm} 25 \%$ iger Salzsăure $1 / 2$ Stunde gekocht, mit Mononatriumkarbonat neutralisiert und mit $25 \mathrm{ccm} n / 10 J o d$ versetzt:

n/10 Thiosulfatverbrauch n/10 Jodverbrauch

$$
18,85 \mathrm{~cm} \quad 6,15 \mathrm{ccm} \quad 87,56 \% \text {. }
$$

8. $20 \mathrm{ccm}$ mit $10 \mathrm{ccm} 25 \%$ iger Salzsäure 1 Stunde gekocht und wie oben behandelt:

$$
\begin{array}{llr}
18,1 \mathrm{ccm} & 6,9 \mathrm{ccm} & 98,24 \% \\
17,91 \text { n } & 7,09 \text { n } & 100,95 \pi
\end{array}
$$

Mit den letzten zwei Versuchen waren also die richtigen Arbeitsbedingungen getroffen worden. Wie ersichtlich, leistet im vorliegenden Falle Salzsäure als Hydratisierungsmittel bessere Dienste, wie die allgemeiner übliche Schwefelsäure. 


\section{Phosphatische Sãure.}

60,205 g obiger phosphatischer Säure ans der keine Krystallisation von unterphosphorsaurem Salz erbalten werden konnten, wurden zu $500 \mathrm{ccm}$ mit Wasser verdünnt, um damit eine Bestimmung der unterphosphorigen und phosphorigen Säure vorzunehmen.

$2 \mathrm{ccm}$ (einem Gewicht von $0,2408 \mathrm{~g}$ entsprechend) wurden mit chlorsaurem Kalium und Salzsăure oxydiert und darin die Totalphosphorsăure bestimmt. Dabei wurden erhalten:

$$
\left.\begin{array}{l}
0,2439 \mathrm{~g} \\
0,2433
\end{array}\right\} \text { im Mittel 0,2436 } \mathrm{g} \mathrm{Mg}_{2} \mathrm{P}_{2} \mathrm{O}_{7} \text {. }
$$

Aus ab initio vorhandener Phosphorsaure wurde an Magnesiumpyrophosphat pro $2 \mathrm{ccm}$ erhalten:

$$
\left.\begin{array}{l}
0,1519 \mathrm{~g} \\
0,1521
\end{array}\right\} \text { im Mittel } 0,1520 \mathrm{~g} .
$$

Die Differenz ergiebt diejenige Gewichtsmenge an Magnesiumpjyrophosphat, welche phosphoriger und unterphosphoriger Säure entstammt.

Für die jodometrische Untersuchung standen $2 \mathrm{~cm} \mathrm{mit} 25 \mathrm{ccm}$ n/10 Jodlösung and $5 \mathrm{ccm}$ verdünnter Schwefelsäure 15 Stunden:

$\begin{array}{cc}\text { n/10 Thiosulfatverbrauch } & \text { Jodverbrauch } \\ \text { 1. } 22,70 \mathrm{ccm} & 2,30 \mathrm{ccm} \\ \text { 2. } 22,35 n & 2,65 n \\ \text { 3. } 22,000 & 2,50 ~\end{array}$

im Mittel 2,48 ccm $=0,0041 \mathrm{~g} \mathrm{H}_{8} \mathrm{PO}_{2}$ entsprechend 0,0068 $\mathrm{g} \mathrm{Mg}_{8} \mathrm{P}_{2} \mathrm{O}_{7}$.

Mit Mononatriumkarbonat neutral gemacht und 1 Stunde gestanden:

$\begin{array}{cc}\mathrm{n} / 10 \text { Thiosulfatrerbrauch } & \text { Jodverbrauch } \\ 1.32,20 \mathrm{ccm} & 17,80 \mathrm{ccm} \\ \text { 2. } 32,45 " & 17,55 " \\ \text { 3. } 32,17 n & 17,83 "\end{array}$

Im Mittel $17,72 \mathrm{ccm}$.

Die Differenz zwischen

$$
\begin{aligned}
& 17,72 \mathrm{ccm} \\
& \text { und } 2,48 \text {, } \\
& 15,24 \mathrm{ccm} \text { Jod für } \mathrm{H}_{8} \mathrm{PO}_{8} \\
& \text { ergiebt } 0,0624 \mathrm{~g} \text {, } \\
& \text { entsprechend } 0,0845_{n} \quad \mathrm{Mg}_{8} \mathrm{P}_{2} \mathrm{O}_{7} \\
& 0,0845 \text { g Mg }_{2} \mathrm{P}_{2} \mathrm{O}_{7} \\
& +\frac{0,0068 \eta n}{0,0913 \text { g } \mathrm{Mg}_{2} \mathrm{P}_{2} \mathrm{O}_{7}}
\end{aligned}
$$


Gravimetrisch waren $0,0916 \mathrm{~g} \mathrm{Mg}_{2} \mathrm{P}_{2} \mathrm{O}_{7}$ für $\mathrm{H}_{8} \mathrm{PO}_{2}+\mathrm{H}_{8} \mathrm{PO}_{8}$ gefunden worden, also auf $0,4 \%$ übereinstimmend. Daraus kann geschlossen werden, dass auch das jodometrisch ermittelte Quantitätsverhăltnis zwischen phosphoriger und unterphosphoriger Säure mit befriedigender Annaherung der Wirklichkeit entsprechen muss.

Es enthielten demzufolge $100 \mathrm{~g}$ jener phosphatischen Săure:

$$
\begin{gathered}
55,73 \% \mathrm{H}_{3} \mathrm{PO}_{4} \\
25,91 \cong \mathrm{H}_{8} \mathrm{PO}_{8} \\
1,70 \cong \mathrm{H}_{3} \mathrm{PO}_{2} \\
16,66 ” \mathrm{H}_{2} \mathrm{O} .
\end{gathered}
$$

Chem. Univers. -Laborat. (Phil. Abt.), Freiburg i. B.

\title{
Ueber Darstellung, Eigenschaften und Entschwefelung des Aethylenthioharnstoffes.
}

\author{
Von Apotheker Dr. H. Klut.
}

(Eingegangen den 17. XI. 1902.)

Den Aethylenthioharnstoff, welcher folgende Konstitution besitzt:

$$
\stackrel{\mathrm{CH}_{2}-\mathrm{NH}_{\mathrm{CH}}}{\mathrm{CH}_{2}-\mathrm{NH}}>\mathrm{CS}
$$

stellte zuerst A. W. Hofmann') durch Einwirkung von Schwefelkohlenstoff auf reines Aethylendiamin in alkoholischer Lösung dar. W. Schacht ${ }^{2}$ ) unterzog später das von Hofmann angegebene Verfahren zur Darstellung dieses Sulfoharnstoffes einer eingehenden Prufung, und bestätigte im allgemeinen die von letzterem gemachten Angaben. Nur fand er den Schmelzpunkt bei $197^{\circ}$, wăhrend Hofmann denselben bei $194^{\circ}$ ermittelte.

Da reines Aethylendiamin jedoch ein ziemlich kostspieliges Präparat ist, kann man vorteilhafter, wie folgt, obigen Harnstoff gewinnen.

Als Ausgangsmaterial dient hierbei das im Handel erhältliche Aethylendiamin in $10 \%$ wåsseriger Lösung, welche eine stark alkalische, schwach gelbliche Flüssigkeit bildet. Zwecks Entfernung des darin stets in geringer Menge vorhandenen Ammoniaks empfiehlt es sich, letzteres durch lăngeres Erhitzen am Rückflusskühler zu entfernen. Die davon befreite Base verwandelt man nun am besten durch Zusatz

1) Berichte d. d. chem. Ges. 5, 242.

ग) Archiv d. Pharm. 1897, 235, S. 441. 